\title{
The study of premature canities and its association in tertiary care hospital
}

\author{
Pavani $\mathrm{P}^{1^{*}}$, Madhavi Latha $\mathrm{M}^{2}$, Praveena $\mathrm{T}^{3}$ \\ ${ }^{1}$ Post graduate, ${ }^{2}$ Professor, ${ }^{3}$ Associate professor, Department of DVL, Santhiram Medical College and General Hospital, NH-40, Andhra \\ Pradesh, INDIA. \\ Email: perumallapavani189@gmail.com
}

\section{$\underline{\text { Abstract }}$}

\begin{abstract}
Background: Canities, or hair graying, is a process of chronological aging and occurs regardless of gender or race. The temples usually show graying first, followed by the crown and later to the scalp's occipital area. Biotin, zinc, and calcium pantothenate commonly used for Prematurecanities. Aim: To study premature greying and its association with other diseases, measure the severity of greying by Graying Severity Score (GSS), and know the efficacy of biotin, zinc, and calcium pantothenate in Prematurecanities. Methods: This study consists of 50 patients presenting with premature canities from September 2019 to September 2020 to the department of DVL, OPD; Santhiram Medical College and General Hospital, Nandyal. A detailed history was taken in a preformed proforma. A thorough scalp examination was done with trichoscopy and took digital photographs. Calculated the GSS and treated with the combination of biotin, zinc, and calciumpantothenate. Results: The earliest age of onset recorded was eight years. Positive family history of was seen in $6(12 \%)$ cases. Smoking was seen in 2(4\%) cases. Association with other diseases were seen as Anaemia in $17(34 \%)$ cases, Alopecia areata in 7(14\%) cases, Thyroid disorder in 4(8\%) cases, Vitiligo in 4(8\%) cases, Atopic dermatitis in $4(8 \%)$ cases. The temporal regions of the scalp were most commonly involved, followed by the frontal area. $8(16 \%)$ subjects had mild GSS I, and they showed moderate efficacy, whereas 19(38\%) had moderate GSS II and showed mild efficacy, but $23(46 \%)$ hadsevere GSS III and showed no efficacy with a combination of biotin, zinc and calcium pantothenate treatment. Conclusion: Premature canities is a significant trichological disorder in India. The most frequent associations observed wereVitiligo, Thyroid disorder, Alopecia areata, Anaemia, smoking. We recommend the Graying Severity Scoring system's application and utility, and essential investigations to know the association of Premature canities with other disorders. Therapy with a combination of biotin, zinc, and calcium pantothenate showed no significant efficacy. The future of treatment options for premature canities lies with targeting genes and proteins involved in hair follicle melanocyte. Keywords: Canities, Graying severity score, Biotin, Zinc, Calcium pantothenate.
\end{abstract}

\section{*Address for Correspondence:}

Dr Pavani.P, Department of DVL, Santhiram Medical College and General Hospital, NH 40, Nandyal, Andhra Pradesh. India.

Email: perumallapavani189@gmail.com

Received Date: 02/08/2020 Revised Date: 11/09/2020 Accepted Date: 27/10/2020

DOI: https://doi.org/10.26611/10211632

This work is licensed under a Creative Commons Attribution-NonCommercial 4.0 International License. (oc)) EY-NC

\begin{tabular}{|l|l|}
\hline \multicolumn{2}{|c|}{ Access this article online } \\
\hline Quick Response Code: & Website: \\
\hline & www.medpulse.in \\
& \\
\hline
\end{tabular}

\section{INTRODUCTION}

Canities, or Hair graying, is a process of chronological aging and occurs regardless of gender or race, which occurs before 20 years in Whites, before 25 years in Asians, and before 30 years in Africans. ${ }^{3}$ Premature canities occur in association with organ-specific autoimmune disorders like Vitiligo, Addison's disease, Graves disease, Pernicious anemia, Hyper- or Hypothyroidism, Progeria, Pangeria, and Atopic diathesis. ${ }^{1,5}$ Reversible hypopigmentation of hair occurs in association with nutritional deficiencies like chronic protein loss (due to kwashiorkor, nephrosis, celiac disease), severe iron deficiency, and copper deficiency. ${ }^{1}$ Other causes include smoking, stress, administration of certain drugs including chloroquine, chemicals like dithranol, topically applied agents, HIV infection, Cystic fibrosis, Hodgkin's lymphoma1Graying usually begins at the temples, then in the sideburns, later spread to the vertex 
and, lastly, affects the occiput. Women typically start graying around the perimeter of the hairline. ${ }^{1}$ Biotin triggers hair growth and treats hair loss related disorders. It also strengthens and thickens the hair. It can reverse the premature greying of hair. Zinc plays a significant role in melanogenesis by its catalytic function in the synthesis of 5,6-dihydroxindole derivatives and inhibits the activity of tyrosinase and glutathione reductase in vitro, enhances the activity of dopachrometautomerase, and has agonistic effects on melanocortin receptor signaling. Because of its anti-oxidative property, zinc protects the melanocytes from free-radical damage. So, zinc deficiency adversely affects melanogenesis. Pantothenic acid (Vitamin B5) is another nutrient to influence hair growth and diameter. It protects the cells against peroxidative damage by increasing the level of glutathione ${ }^{13}$.

\section{MATERIALS AND METHODS}

An observational study was conducted in patients presenting with Prematurecanities to the outpatient department of DVL of Santhiram Medical College and General Hospital, Nandyal, from September 2019 to September 2020. Patients willing to give consent were included in the study were clinically examined and did trichoscopy. Un-cooperative patients or patients who cannot understand the protocol and provide informed consent or patients with unrealistic expectations and patients already on treatment were excluded. Patients were advised for follow-up visits once monthly and did a final assessment by the end of the 6th month. The institutional committee has approved this study and written informed consent was taken from all study subjects. A detailed history of onset, pattern, progression, and family history of graying was recorded on a predesigned proforma and taken clinical photographs. The entire scalp surface was divided into five zones: frontal region, vertex, right and left temporal areas, and the occipital. In each of these zones, areas showing maximum graying were identified on visual examination. A $1 \mathrm{~cm} 2$ place was marked with a skin marker, and the hair within this square was cropped to approximately $1 \mathrm{~mm}$ above the scalp surface. These five squares were then photographed and projected on the computer screen to count the white and black hair numbers.

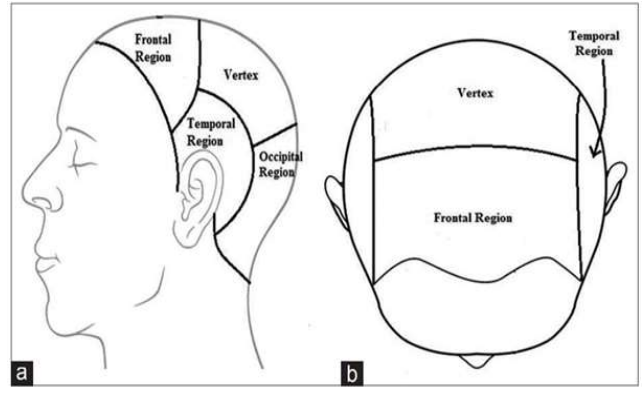

Figure 1: The 5 representative zones of scalp (a) side profile (b) top view

Based on the hair count, assigned a score according to the percentage of gray hair in each square. This was calculated and scored as: Score 1 (assigned to under 10\% gray hair/cm2); Score $2(10 \%-30 \%$ gray hair/cm2); and Score 3 (more than $30 \%$ grey hair $/ \mathrm{cm} 2$ ) We calculated the Greying Severity Score (GSS) for each patient by taking a sum of the five representative sites' scores. Thus the maximum attainable score for a patient was $15(3 \times 5)$. We graded the objective scores as Mild (a score of $0-5$ ), Moderate(score of 6-10), and Severe (score of 11-15).

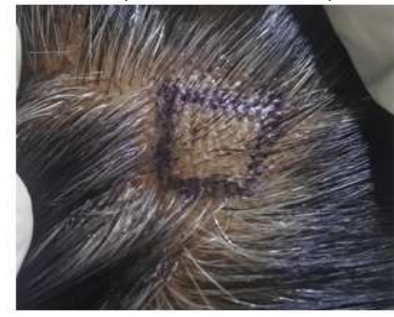

a

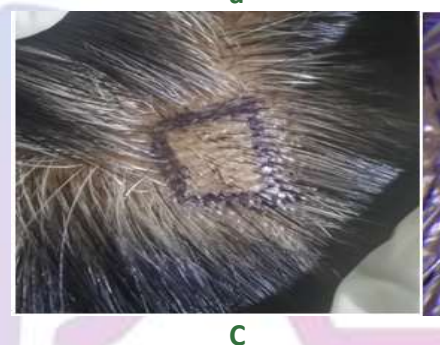

C

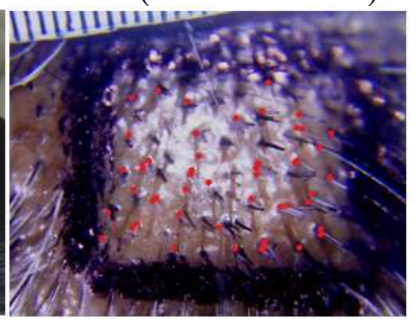

b

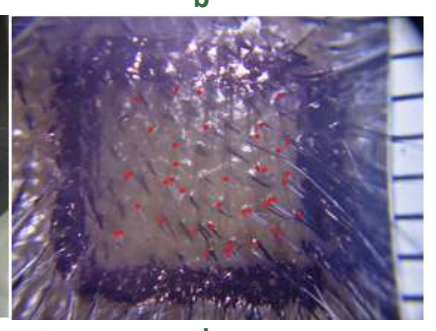

d
Figure 2: ( $a$ and $c$ ) Clinical photograph of study subjects demonstrating a $1 \mathrm{~cm} 2$ area with maximum graying in the vertex region. ( $b$ and d) Magnified view of the photograph on left (Grey hair represented by red dots). The first case(a) had moderate premature canities with graying severity score (GSS) $=4$ and the second case (c) had severe premature canities with GSS $=6$.

\section{RESULTS}

This study sample consisted of 50 patients, out of which 26 were males, and 24 were females, where the majority belonging to the age group of 10-20 years. The earliest age of onset recorded was eight years. Six cases have a positive family history, and two patients were smokers. Premature canities were associated with other diseases as Anaemia in 17 cases, Thyroid disorder in 8 cases, Alopecia areata in 7 cases, Vitiligo in 4 cases, Atopic dermatitis in 4 cases. The scalp's temporal regions were most commonly involved, followed by the frontal, occipital, and vertex region. $8(16 \%$ ) subjects had mild GSS I, 19(38\%) had moderate GSS II, and23(46\%) had severe GSS III. Therapy with a combination of biotin, zinc, and calcium pantothenate showed moderate efficacy in GSS I. Out of 8, 4 cases showed improvement. Mild effectiveness in GSS II, out of 19 , only five patients showed improvement, and shifted 
from GSS II to GSS I. This therapy showed no effect on GSS III.

Table 1: AGE DISTRIBUTION

\begin{tabular}{ccc}
\hline Age & Number & Distribution \\
\hline $1-10$ yrs & 9 & $18 \%$ \\
$11-20 y r s$ & 27 & $54 \%$ \\
$21-30$ yrs & 14 & $28 \%$ \\
\hline
\end{tabular}

Table 2: SEX DISTRIBUTION

\begin{tabular}{ccc}
\hline Gender & Number & Percentage \\
\hline Males & 26 & $52 \%$ \\
Females & 24 & $48 \%$ \\
\hline
\end{tabular}

\begin{tabular}{ccc}
\multicolumn{3}{c}{ Table 3: DISTRIBUTION OF CANITIES } \\
\hline Site & Number & Percentage \\
\hline Frontal & 14 & $28 \%$ \\
Vertex & 6 & $12 \%$ \\
Temporal & 21 & $42 \%$ \\
Occipital & 9 & $18 \%$ \\
\hline
\end{tabular}

Table 4: ASSOCIATION WITH FAMILY HISTORY

\begin{tabular}{ccc}
\hline & Number & Percentage \\
\hline Present & 6 & $12 \%$ \\
Absent & 44 & $88 \%$ \\
\hline
\end{tabular}

Table 5: ASSOCIATION WITH SMOKING

\begin{tabular}{ccc}
\hline & Number & Percentage \\
\hline Present & 2 & $4 \%$ \\
Absent & 48 & $96 \%$
\end{tabular}

Table 6: ASSOCIATION WITH ANAEMIA

\begin{tabular}{ccc}
\hline & Number & Percentage \\
\hline Present & 17 & $34 \%$ \\
Absent & 33 & $66 \%$ \\
\hline
\end{tabular}

Table 7: ASSOCIATION WITH THYROID DISORDER

\begin{tabular}{ccc}
\hline & Number & Percentage \\
\hline Present & 4 & $8 \%$ \\
Absent & 46 & $92 \%$ \\
\hline
\end{tabular}

Table 8: ASSOCIATION WITH OTHER DISEASES

\begin{tabular}{ccc}
\hline & Number & Percentage \\
\hline Vitiligo & 4 & $8 \%$ \\
Alopecia areata & 7 & $14 \%$ \\
Atopic dermatitis & 5 & $10 \%$ \\
No association & 34 & $68 \%$ \\
\hline
\end{tabular}

GRAYING SEVERITY SCORE: BEFORE

TREATMENT

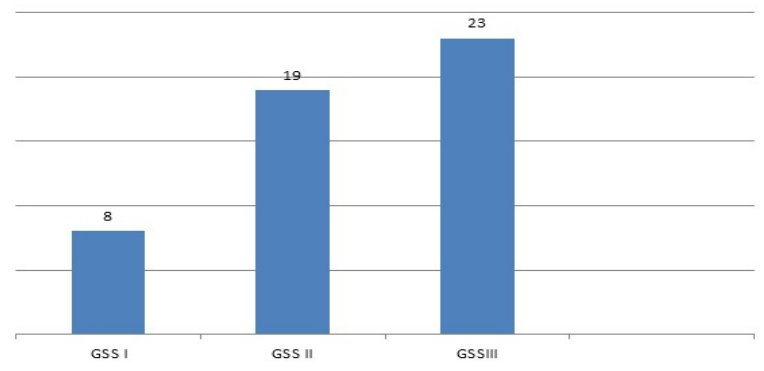

Figure 3: Bar graph representing the graying severity score of premature canities on $\mathrm{X}$-axis and the number of cases with the respective severity score on $\mathrm{Y}$-axis

GRAYING SEVERITY SCORE: AFTER 6 MONTHS OF TREATMENT WITH CONBINATION OF BIOTIN, ZINC AND CALCIUM PANTOTHENATE

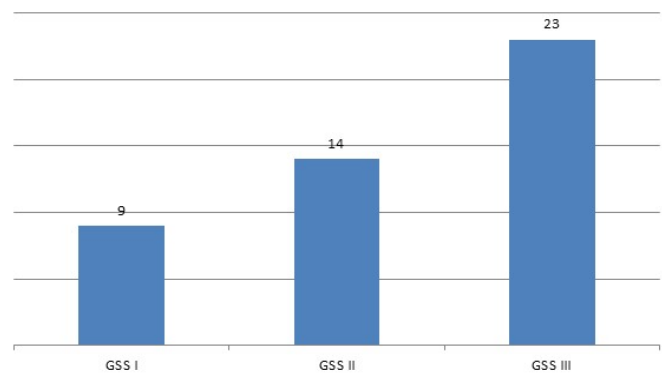

Figure 4: Bar graph representing the graying severity score of premature canities on $\mathrm{X}$-axis and the number of cases with the respective severity score on $\mathrm{Y}$-axis

\section{CLINICAL PHOTOGRAPHS}

GSS I:

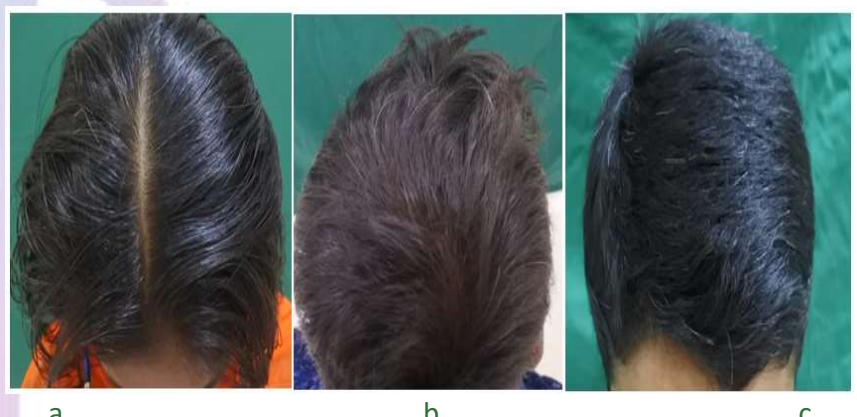

a

GSS II:

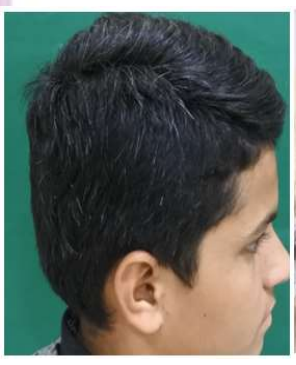

a

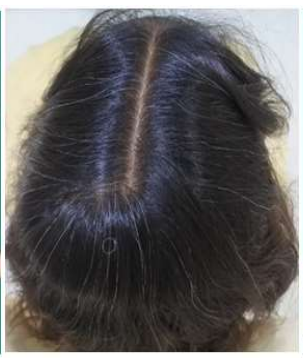

b

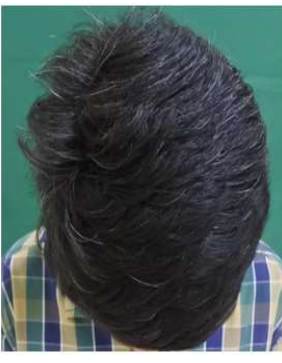

C

\section{GSS III:}




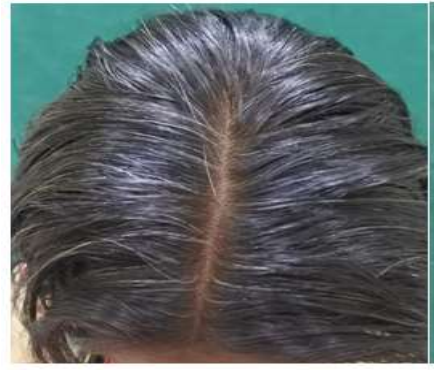

a

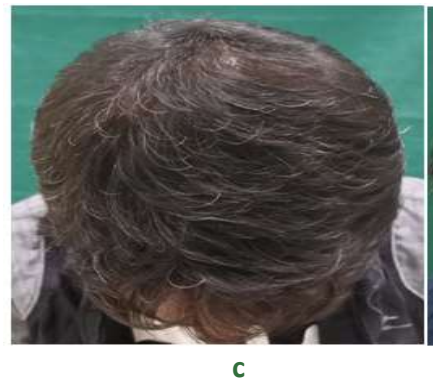

C

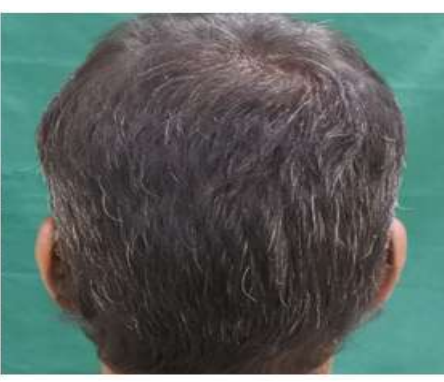

b

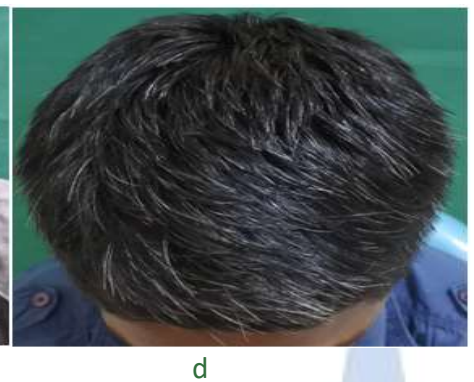

\section{DISCUSSION}

Skin and hair play an essential role in human outlook. The hair's length and color are of immense value to an individual's cosmetic appearance. Aging of hair occurs due to weathering of the hair shaft and aging hair follicle. Weathering of the hair shaft involves the degeneration of hair that progresses from the root to the tip. Aging of hair follicles due to reduced melanocyte function (known as graying). ${ }^{3}$ The primary cause of Prematurecanities is genetic, although autoimmune disorders such as Vitiligo, Pernicious anemia, Werner's syndrome, and Autoimmune thyroid disorders are also causative. Few studies have also reported that ultraviolet light, smoking, drugs, deficiencies of trace elements, and nutritional deficiencies also play a role in Premature canities. ${ }^{3}$ In this study, positive family history of Prematurecanities was reported in $6(12 \%)$ cases, whereas Sonthalia et al. s study reported $21 \%$ of family history. ${ }^{2}$ In this study, most cases noticed the first strands of gray hair in the temporal areas, followed by the frontal region. Even physiological graying also tends to start from the temporal region and sideburns in men and scalp margins in women. While Daulatabad et al.. reported a reverse pattern, i.e., frontal involvement more than temporal and hypothesized that premature canities might represent an entity distinct from chronological graying. ${ }^{2}$ Iron and vitamin B12 insufficiency have a role to play in the pathogenesis of Prematurecanities. This study showed a 34\% association with Anaemia. ${ }^{4}$ Thyroid disorders, including hypo, and hyperthyroidism, thyroiditis, have conventionally been suggested to be associated with Prematurecanities. This study showed an $8 \%$ association with a thyroid disorder. Daulatabad et al. study noticed no association of any thyroid abnormality in their 52 selfreporting patients with premature canities. ${ }^{4}$ Zayed et al. reported a significant association between Prematurecanities and cigarette smoking. This study also reported a $4 \%$ of the association between Premature canities and cigarette smoking. ${ }^{3}$ GSS has the advantage of being an objective, numeric, and reproducible scoring system and objective means of scoring the graying, considering all areas of the scalp. Secondly, the data maintained as a photographic record for subsequent evaluation. Also, this data can be helpful in therapeutic trials with pre- and post-treatment scores. The high level of agreement achieved between two independent investigators' observations suggests a reproducible scoring system. Lastly, the scoring system provides us with an objective measure of the severity of premature canities in the different scalp regions, enabling assessing the clinical pattern and extent of graying. In the present study, the subjects found maximum involvement of the temporal region closely followed by the scalp'sfrontal area. In the future, this scoring system may be extrapolated to assess the extent and severity of physiological canities. ${ }^{5}$ Another scoring system known as the hair whitening score (HWS) has been used in two consecutive studies by Erdoğan and Kocaman et al.. HWS was defined according to the percentage of white hair (HWS 1 (Trace): $<25 \%$; HSW 2 (Mild):25\%-50\%; HSW 3 (Moderate): 50\%-75\%: HSW 4 (Manifest): 75\%-100\%: HSW 5 (Complete): 100\%). As studies were done on older individuals and not on patients with premature canities, this scale may not apply to younger subjects before 20 . Thus according to this scoring, most of the patients would fit into only two categories, mild or moderate, which restricts further evaluation. ${ }^{5}$

\section{CONCLUSION}

The present study reveals that Premature canities is a significant trichological disorder in India. The onset is typically in late childhood or early adolescence, with the temporal and frontal areas being the most commonly involved sites. The most frequent associations observed in this study were Vitiligo, thyroid disorder, alopecia areata, Anaemia, and smoking. Family history also has a relation with premature canities. Due to the lack of a widely recognized standard scoring system for evaluating premature canities, GSS as a novel, numeric, objective, and reproducible tool. The GSS can follow up and assess therapeutic responses that can maintain the data in a photographic record. We recommend further studies on a larger population to optimize this novel scoring system's application and utility. It is necessary to know the association of Prematurecanities with other disorders with essential investigations. Therapy with a combination of biotin, zinc, and calcium pantothenate showed no 
significant efficacy. The future of treatment options for premature canities lies with targeting genes and proteins involved in the hair follicle.

\section{REFERENCES}

1. Pandhi D, Khanna D. Premature graying of hair. Indian JDermatolVenereolLeprol. 2013;79:641-53.

2. Sonthalia S, Priya A, Tobin D J. Demographic Characteristics and Association of Serum Vitamin B12, Ferritin and Thyroid Function with Premature Canities in Indian Patients from an Urban Skin Clinic of North India: A Retrospective Analysis of 71 Cases.Indian J Dermatol. 2017; 62(3): 304-308.

3. Chakrabarty S, Krishnappa P G, Gowda D G, Hiremath J. Factors Associated with Premature Hair Greying in a Young Indian Population. Int J Trichol. 2016;8(1):11-14.

4. Almutairi R T,Dhafiri M S. Premature greying of hair among the population of King Faisal University in AlAhasa, Saudi Arabia: an epidemiological study. 2019.

5. Singal A, Daulatabad D, Grover C.Graying severity score: A useful tool for evaluation of premature canities. Indian Dermatol Online J. 2016; 7(3): 164-167.

6. Knagha A B, Shamim H, Nagaraju U. Premature Graying of Hair: Review with Updates. Int J Trichol. 2018;10:198203.
7. Sharma N, Dogra D.Association of Epidemiological and Biochemical Factors with Premature Graying of Hair: A Case-Control Study. Int J Trichology. 2018; 10(5): 211217

8. Daulatabad D, Grover C, Singal A. Quality of life and psychological impact of premature canities: A study from North India.Pigment int. 2016;3:24-8.

9. McDonough PH, Schwartz RA. Premature hair graying. Cutis. 2012;89(4):161-5.

10. Wood JM, Jimbow K, Boissy RE, Slominski A, Plonka PM, Slawinski J, et al.. What's the use of generating melanin? ExpDermatol 1999;8:153-64

11. Ashraf P, Simanungkalit R, Jusuf N K. Association between premature canities and quality of life at Medan, Indonesia 2017. Bali Med J. 2018;7(2):339-45.

12. Lee W S. Photoaging of Hair.Int J Trichol. 2009;1(2):9499.

13. Siavash M, Tavakoi F, MokhtariF.Comparing the Effects of Zinc Sulfate, Calcium Pantothenate, Their Combination and Minoxidil Solution Regimens on Controlling Hair Loss in Women: A Randomized Controlled Trial. J Res

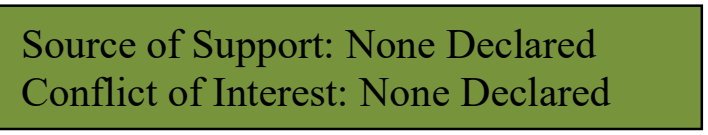

